$\begin{array}{cl}\begin{array}{cl}\text { Revue } \\ \text { de I'histoire }\end{array} & \text { Revue de l'histoire des religions } \\ \text { des religions } & \begin{array}{l}1 \mid 2017 \\ \text { Varia }\end{array}\end{array}$

\title{
François LISSARRAGUE, La cité des satyres. Une anthropologie ludique (Athènes, $\mathrm{VI}^{e}-\mathrm{V}^{e}$ siècles avant J.-C.)
} Paris, Éditions de l’EHESS («L'histoire et ses représentations », 8), 2013

\section{Richard Veymiers}

\section{(2) OpenEdition Journals}

\section{Édition électronique}

URL : http://journals.openedition.org/rhr/8680

DOI : ERREUR PDO dans /localdata/www-bin/Core/Core/Db/Db.class.php L.34 : SQLSTATE[HY000]

[2006] MySQL server has gone away

ISSN : 2105-2573

\section{Éditeur \\ Armand Colin}

\section{Édition imprimée}

Date de publication : 1 mars 2017

Pagination : 150-153

ISBN : 978-2-200-93125-4

ISSN : 0035-1423

\section{Référence électronique}

Richard Veymiers, "François Lissarrague, La cité des satyres. Une anthropologie ludique (Athènes, vi ${ }^{e}$ $v^{e}$ siècles avant J.-C.) 》, Revue de l'histoire des religions [En ligne], 1 | 2017, mis en ligne le 24 mars 2017, consulté le 07 janvier 2021. URL : http://journals.openedition.org/rhr/8680 ; DOI : https://doi.org/ ERREUR PDO dans /localdata/www-bin/Core/Core/Db/Db.class.php L.34 : SQLSTATE[HY000] [2006] MySQL server has gone away

Ce document a été généré automatiquement le 7 janvier 2021.

Tous droits réservés 


\section{François LISSARRAGUE, La cité des satyres. Une anthropologie ludique (Athènes, $\mathrm{VI}^{e}-\mathrm{V}^{e}$ siècles avant J.-C.)}

Paris, Éditions de l'EHESS (« L'histoire et ses représentations », 8), 2013

\section{Richard Veymiers}

\section{RÉFÉRENCE}

François LISSARRAGUE, La cité des satyres. Une anthropologie ludique (Athènes, $\mathrm{VI}^{e}-\mathrm{V}^{e}$ siècles avant J.-C.), Paris, Éditions de l'EHESS («L'histoire et ses représentations », 8), 2013, 21,5 cm, 327 p., $35 €$, ISBN 978-2-7132-2384-6.

1 Éminent spécialiste de l'image et de son fonctionnement dans les sociétés antiques, F.L. explore, à travers l'étude de la production céramique attique et le jeu de ses compositions, un univers de fantaisie, celui des satyres grecs, ces êtres hybrides, à la fois chevalins et humains, qui relèvent du thiase de Dionysos. Ce monde imaginaire, dont ce bel ouvrage vise à cerner la logique figurative, voit le jour à Athènes, au début $\mathrm{du} \mathrm{VI}^{\mathrm{e}} \mathrm{s}$., au moment où se développe la cité. Il va ensuite proliférer sous des formes diversifiées, sous l'action de peintres parfois très inventifs, sur des vases de banquet à figures noires, puis rouges, jusqu'à la fin du $\mathrm{IV}^{\mathrm{e}} \mathrm{s}$.

2 Après avoir posé quelques jalons (1. "Commencements", 11-19), notamment historiographiques, F.L. évoque à côté des images un autre mode d'expression, né à Athènes, faisant intervenir des satyres: le théatre et en particulier le «drame satyrique » qui se développe à partir de 500 au sein du concours des Grandes Dionysies (2. "Tous en scène ", 21-37). D'aucuns ont eu tendance à considérer les images des vases comme des illustrations de ces spectacles qui intègrent un chœur de satyres à des histoires données, comme le Cyclope d'Euripide ou les Limiers de Sophocle. Or les images ne sont nullement dépendantes du théâtre et de ses textes. Elles se suffisent à ellesmêmes. L'activité théâtrale n'est qu'une des références que les peintres se sont 
appropriées parmi les pratiques culturelles qui leur étaient familières. C'est ce que révèle une appréhension globale de cette culture visuelle. En considérant quelque 7000 vases attiques représentant des satyres, sans négliger d'autres supports matériels, comme la petite plastique en bronze et en terre cuite ou la grande statuaire de pierre (auxquels il aurait pu associer la glyptique), F.L. analyse judicieusement les images dans le réseau iconographique auquel elles appartiennent, ce qui lui permet de mieux saisir les mécanismes qui régissent les scènes, et de se rapprocher ainsi des intentions des peintres et des critères qui ont présidé à leurs choix figuratifs.

Cette vue d'ensemble nous fait découvrir un monde imaginaire varié, un répertoire à la fois riche et complexe, où les satyres sont soumis à un large spectre de manipulations possibles. Cette plasticité figurative du motif est amplifiée par la quasi-absence de mythe et de généalogie les concernant, en dehors de deux récits qui se développent toutefois tardivement dans l'iconographie, ceux de Marsyas et de Silène. L'inventivité des peintres se donne ainsi libre cours, comme celle des poètes tragiques d'ailleurs. L'histoire visuelle des satyres est pleine de métamorphoses et de surprises. Pour en rendre compte, F.L. retient une série de thématiques transversales, nourries d'exemples soigneusement choisis, qui caractérisent cet univers figuratif et construisent une définition de cette figure mythologique sans mythe qu'est le satyre.

En grec, "peindre ", c'est aussi " écrire ", d'où cette habitude d'inscrire à côté des figures des noms qui ont un rôle à la fois linguistique et visuel. La production céramique attique fait ainsi connaître quelque 120 noms de satyres, d'abord descriptifs, parlant de ceux qui les portent, puis, à partir du ve s., plus allégoriques (3. « Noms de satyres, le nom ", 39-52). Une onomastique significative qui se décline en diverses catégories, parfois combinées en une même image, pour exprimer autant de facettes du monde des satyres. L'une d'elles recouvre leur anatomie qui fait l'objet d'élaborations diverses modulant leur hybridité (4. « Le corps du satyre », 53-71). Autour du vase, dont ils ornent souvent les points d'articulation, ils apparaissent comme des corps démultipliés, relevant d'un genos masculin, se constituant progressivement en diverses classes d'âges, mais aussi comme des corps mobiles, instables dans leurs proportions, postures et gestes, et comme des corps sensibles, qui hument, écoutent, goûtent et observent. Le trait le plus marquant est toutefois leur ithyphallisme démesuré et exhibé, révélant une indécence, une énergie sexuelle, qu'ils manifestent avec toutes sortes de partenaires érotiques, qui ne sont pas nécessairement des êtres animés (5. «De la sexualité des satyres », 73-96). La bestialité des satyres, qui rappelle celle des centaures - dont ils se distinguent toutefois tant dans leur hybridité que par leur comportement-, leur vaut de nouer des rapports avec diverses espèces animales présentant des caractères singuliers entrant en résonance ou en opposition avec eux (6. "Animalités comparées ", 97-130). Si leur hypersexualité, par exemple, les rapproche des ânes, avec lesquels ils peuvent d'ailleurs s'accoupler, elle les distingue des singes qui affichent une insuffisance trop humaine en la matière.

5 La sociabilité des satyres s'exprime avant tout dans l'univers festif du banquet où ils se comportent en échansons, voire en parfaits cômastes, autour de Dionysos (7. « Le vin des satyres ", 131-147). L'imaginaire du vin et de la vigne est ainsi omniprésent dans le répertoire de ces vases destinés aux symposia. Le monde des satyres est fait d'abondance et de légèreté, et le vin n'est jamais loin, tout comme la musique et la danse (8. "Musique et danse », 148-173). Déformant le visage, l'aulos convient bien aux satyres, qui jouent aussi de la lyre et de la cithare dans le thiase de Dionysos. La dynamique de 
la musique enclenche généralement une danse, exécutée par un chœur à la gestuelle expressive, qui reste toutefois indéfinie, à l'exception de la pyrrhique, reconnaissable aux armes portées par les danseurs. Les satyres prennent part à des formes de sociabilité plus larges, faisant la guerre aux côtés de Dionysos dans son combat contre les géants (9. "Dionysos s'en va-t-en guerre», 175-189). Combattants marginaux, souvent armés à la légère, mais n'ayant garde d'oublier le vin ou leur lubricité, ils peuvent même servir d'épisèmes, marquant de leur empreinte le bouclier de véritables guerriers. La communauté dionysiaque paraît parfois fonctionner comme une cité, dont les satyres investissent les espaces (10. "Satyres dans la cité », 191-215). On les voit participer à des fêtes et rituels dionysiaques, poser des actes religieux envers les dieux. Vêtus en citoyens, ils apparaissent au sein de l'espace civique, intégrant même le cadre domestique, ou jouant comme des enfants. Mis au travail, ils agissent en artisans ou en paysans et sont alors plus proches de l'esclave que de l'homme libre. Dans le monde des dieux, ils interfèrent sous des formes multiples avec diverses puissances, faisant souvent office de figures intermédiaires, au sein d'assemblées ou de banquets par exemple, conformément à leur rôle auprès de Dionysos, dont ils peuvent même être les éducateurs (11. "L'Olympe des satyres ", 217-241). Concurrençant parfois les amours des dieux dans des épisodes mettant en jeu leur sexualité, comme celui de Poséidon poursuivant Amymonè, ils cèdent progressivement le pas à Éros, qui finit par assurer la dimension érotique lorsque se recoupent les domaines de Dionysos et d'Aphrodite.

Ce voyage au pays des satyres que F.L. nous fait découvrir par ces thèmes caractéristiques recouvre une histoire visuelle de plus de deux siècles, au cours desquels le répertoire s'est transformé, grâce à des peintres - comme celui de Géras dont la créativité a pu être en partie stimulée par le développement du drame satyrique. On voit ainsi apparaître au tournant $d u v^{e} s$. des bébés satyres, des adolescents et des vieillards, alors qu'auparavant, en figures noires, leur apparence était essentiellement celle d'un adulte. De même, à partir de 500, les ménades cessent d'être consentantes, fuyant davantage les assauts des satyres. Les innovations s'amplifient après 450 avec la multiplication des satyres citadins, l'atténuation de leur ithyphallisme, ou leur association à Éros. Tout se passe comme si le satyre "se civilisait ».

7 Au-delà de ces évolutions, il y a au cœur de ce répertoire satyresque tout un travail à l'œuvre sur la culture athénienne que la perspective anthropologique de l'étude fait très clairement ressortir. On ne peut comprendre le fonctionnement de ces images qu'en les analysant dans leur contexte d'utilisation, qu'en les pensant dans leur propre

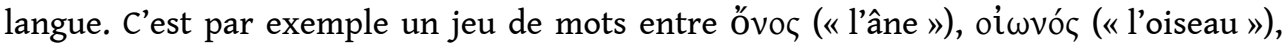
voire oĩvoৎ (« le vin ») qui éclaire l'image d'un hybride mi-âne, mi-oiseau, effrayant un satyre sur une oenochoè (fig. 89). L'instabilité, la malléabilité des satyres permet aux peintres de jouer avec les normes et les pratiques civiques des Athéniens qu'ils nous permettent paradoxalement de mieux saisir. En recourant à des jeux d'imitation, d'inversion ou de substitution, soit diverses stratégies visuelles, ils introduisent délibérément des interférences, des décalages qui créent des courts-circuits, des effets de surprise, déclenchant le sourire ou le rire. Des images, comme celles de satyres s'accouplant avec une amphore (fig. 57), servant de monture à un âne (fig. 81), combattant avec une outre et un rython (fig. 155) ou s'approchant de la toison à la place de Jason (fig. 14), sont autant de détournements, de parodies, de plaisanteries visuelles qui mettent en tension le monde du thiase et celui des hommes. Les satyres ne 
suivent pas le modèle du $\kappa \alpha \lambda o ́ \varsigma \kappa \alpha$ ỏ $\alpha \theta o ́ \varsigma$, de ce citoyen idéal de la cité d'Athènes à la fois «bel et bon». Certes, leur imaginaire n'est pas seulement un monde à l'envers. C'est aussi un monde à souhait, où tout est facile et possible, parfaitement adapté à l'univers des symposia. Figures de l'excès, les satyres sont bons à montrer, en image comme au théâtre. Sorte de miroir déformant de la culture humaine, ils dessinent une anthropologie décalée de la cité, invitant leurs spectateurs à prendre leurs distances, à ne pas sombrer dans les mêmes excès. Comme l'écrit F.L. au terme de cette brillante et foisonnante étude, en rappelant la plaisanterie d'Alcibiade au Banquet de Platon (Coda "Socrate, le satyre », 243-245), « nul ne se veut satyre, sinon au banquet ».

\section{AUTEURS}

\section{RICHARD VEYMIERS}

Université de Liège. 